\title{
Face illumination processing using nonlinear dynamic range adjustment and gradientfaces
}

\author{
Zhi-Jun Yang, Xiang-Fei Nie ${ }^{\dagger}$, Xue He and Wen-Yi Xiong \\ College of Electronic and Information Engineering, \\ Chongqing Three Gorges University, Wanzhou, Chongqing, 404000, China \\ E-mail: niexf@tom.com
}

\begin{abstract}
In this paper, a novel illumination processing approach based on nonlinear dynamic range adjustment and gradientfaces is discussed. Firstly, the grayscale of face image is adjusted by nonlinear dynamic range adjustment using hyperbolic sine function in logarithm domain. After finishing the adjustment, gradientfaces is used to enhance the high frequency component of face image and extract distinguishing facial feature. Secondly, the data dimensionality is reduced by principal component analysis (PCA), the nearest neighbourhood-based classifier based on cosine distance is adopted for face classification. The experiment results on Yale-B frontal face database demonstrates that the average face recognition rate of our technique can reach to $99.74 \%$. Therefore, it is insensitive to different lighting resources.
\end{abstract}

Keywords: Illumination Processing; Nonlinear Dynamic Range Adjustment Gradientfaces; Face Recognition.

\section{Guidelines}

It is a well-known fact that one of the challenge of face recognition is illumination problem [1]. Same faces under the condition with different lighting holds bigger variations compared with different faces under the same lighting circumstance. Thus, there are a variety of algorithms to deal with the problem above. In general, those algorithms can be divided into three main categories [2]. The first sort is to extract the illumination invariant features or illumination insensitive measures for face images. In ref. [3], the author has combined the image normalisation and illumination invariant descriptor to attain the illumination insensitive facial feature. In ref. [4], the author has provided an illumination-invariant face recognition method called adaptive homomorphic eight local directional pattern. However, the calculation of this algorithm is complicated. The second approach is image pre-processing or illumination normalization face images under varying lighting condition. For instance, histogram equalization (HE), gamma correction, logarithm transform, and so on, are widely used for image pre-processing or illumination normalization. This 
approach can alleviate the effect of lighting change to some extent. However, it is difficult to restore local facial feature with extreme lighting. The third approach, which has handled the illumination problem by constructing face model. The main principle of this approach is to research the level of lighting change of face images under appropriate subspace or manifold. In ref. [5], the author shows a novel framework for 3D face reconstruction from a single 2D face image. In ref. [6], the author demonstrates the application of the facial symmetry assumption in 3D face reconstruction. Yet this approach needs mass face images with different illumination condition as test samples, which has limitation for actual application.

To the best of our knowledge, the ideal illumination preprocessing technique is extracting illumination insensitive facial feature. In this paper, we are extracting distinguishable image feature using gradientfaces to study the relationship between adjacent pixels after nonlinear dynamic range adjustment.

The rest of this correspondence is organized as follows. In section II, the logarithm transform, nonlinear dynamic range adjustment and gradientfaces are described. The face database, parameter selection and experimental results are presented in section III. Finally, the conclusion is given in section IV.

\section{Methodologies}

In this article, we present an illumination insensitive facial feature extraction method for face recognition under varying lighting condition. Fig. 1 shows the flow diagram of the illumination pre-processing.

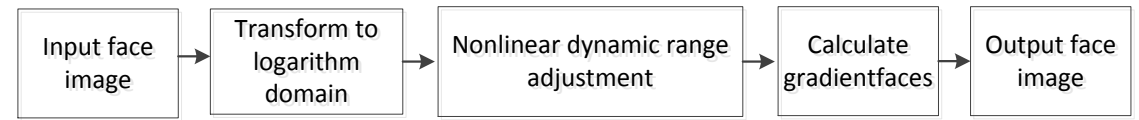

Fig. 1. The flow diagram of illumination processing.

\subsection{Nonlinear dynamic range adjustment}

The principle of nonlinear dynamic range adjustment to image using the function of $\sinh (x)$ is shown in Fig.2. Taking the procedure from the signature was received by eyes to image was developed in brain into account, which is similar to logarithm mapping, nonlinear dynamic range adjustment using logarithmic transform are realized.

Calculation formula of the nonlinear dynamic range adjustment is shown as follows:

$$
I_{2}(i, j)=c \lg \left(1+\sinh \left(I_{1}(i, j)\right)\right)(i=1,2, \cdots, m ; j=1,2, \cdots n)
$$


Where $I_{1}(i, j)$ is original image. $I_{2}(i, j)$ is face image after adjusted by nonlinear dynamic range adjustment. $c$ is a constant. $\lg (x)$ is logarithm function. The size of face image is $m \times n$.

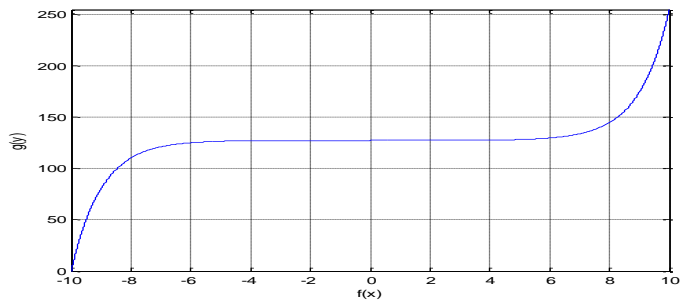

Fig. 2. The mapping relation of nonlinear dynamic range adjustment

\subsection{Gradientfaces}

The main idea of gradientfaces [7] is as following:

Considering two neighboring pixel point $(x, y)$ and $(x+\Delta x, y)$, we have

$$
I(x+\Delta x, y)=R(x+\Delta x, y) L(x+\Delta x, y)
$$

Subtracting Eq. (1) from Eq. (4), we obtain

$$
I(x+\Delta x, y)-I(x, y)=R(x+\Delta x, y) L(x+\Delta x, y)-R(x, y) L(x, y)
$$

Based on the common assumption, which means $L$ is approximately smooth and changes slowly, thus we have

$$
\begin{aligned}
& I(x+\Delta x, y)-I(x, y)=R(x+\Delta x, y) L(x+\Delta x, y)-R(x, y) L(x, y) \\
& \approx(R(x+\Delta x, y)-R(x, y)) L(x, y)
\end{aligned}
$$

Taking the limitation of Eq. (6), we can obtain

$$
\frac{\partial I(x, y)}{\partial x} \approx L(x, y) \frac{\partial R(x, y)}{\partial x}
$$

Similarly we have

$$
\frac{\partial I(x, y)}{\partial y} \approx L(x, y) \frac{\partial R(x, y)}{\partial y}
$$

Dividing (7) by (8), we get

$$
\frac{\partial I(x, y)}{\partial y} / \frac{\partial I(x, y)}{\partial x} \approx \frac{\partial R(x, y)}{\partial y} / \frac{\partial R(x, y)}{\partial x}
$$

According to the illumination model, $R(x, y)$ is considered to be illumination insensitive measure, so as to the radio the gradient of $y$ direction $(\partial I(x, y) / \partial y)$ 
and the gradient of $x$ direction $(\partial I(x, y) / \partial x)$. Thus, in this paper, we explore the illumination insensitive facial feature through $I_{y} / I_{x}$.

$I$ be an image under varying lighting, then gradientfaces $(\mathrm{G})$ of image $I$ can be defined as

$$
G=\arctan \left(I_{y} / I_{x}\right), G \in[-\pi, \pi]
$$

Where $I_{y}$ and $I_{x}$ are the gradient of $y$ and $x$ direction, respectively.

To compute the gradient stably, we smooth face images with Gaussian kernel function firstly.

$$
I^{\prime}=I * G(x, y, \sigma)
$$

The Gaussian kernel function is expressed as follows:

$$
G(x, y, \sigma)=\frac{1}{2 \pi \sigma^{2}} \exp \left(-\frac{x^{2}+y^{2}}{2 \sigma^{2}}\right)
$$

Where ${ }^{\sigma}$ is the standard deviation.

\section{Experiment}

\subsection{Parameter selection}

The mean and variance of face image are 0 and 1after illumination processing. In the stage of image classification, the principal component analysis (PCA) is adopted to extract facial feature, in which the feature vectors are 50. The classifier is nearest neighborhood classifier based on cosine distance. When we are choosing different standard deviation $\sigma$ of the Gaussian kernel function in Eq. (10), the degree of smooth to face images is differ, which will affect the extraction of the facial feature and the rate of face recognition. Consequently, we need to do exploration with different $\sigma$ to find the best result of the face recognition. Ultimately, the parameter $\sigma$ on Yale-B database are $0.31,0.41,0.3$, 0.3 and 0.1 with different training subset in Table 1 , respectively. 
Table. 1. Experiment result on Yale-B

\begin{tabular}{cc|c|c|c|c}
\hline \multirow{2}{*}{ Train Set } & \multicolumn{5}{c}{ Test Set } \\
\cline { 2 - 6 } & \multicolumn{5}{c}{ Face regnition rate $(\%)$} \\
\hline \multirow{2}{*}{ subset1 } & subset2 & subset3 & subset4 & subset5 & average \\
\cline { 2 - 6 } & 100 & 100 & 99.29 & 99.47 & 99.65 \\
\hline \multirow{2}{*}{ subset2 } & subset1 & subset3 & subset4 & subset5 & average \\
\cline { 2 - 6 } & 100 & 100 & 99.29 & 97.89 & 99.04 \\
\hline \multirow{2}{*}{ subset3 } & subset1 & subset2 & subset4 & subset5 & average \\
\cline { 2 - 6 } & 100 & 100 & 100 & 100 & 100 \\
\hline \multirow{2}{*}{ subset4 } & subset1 & subset2 & subset3 & subset5 & average \\
\cline { 2 - 6 } & 100 & 100 & 100 & 100 & 100 \\
\hline \multirow{2}{*}{ subset5 } & subset1 & subset2 & subset3 & subset4 & average \\
\cline { 2 - 6 } & 100 & 100 & 100 & 100 & 100 \\
\hline
\end{tabular}

\subsection{Experiment results}

Fig.3 shows the result of original images handled by different methods. As can be seen, the gray level range of face image is changed greatly after finishing nonlinear dynamic range adjustment. The gray value of face image was made dynamic range adjustment by a smooth project curve, which renders the change of the gray value more smoother and the range of change of the greyscale of some region we concerned amplify. After nonlinear dynamic range adjustment, the detail of image is clearer and the contrast of the whole image enlarged. We draw a picture from them that the important facial features, such as eyes, noses, mouse and other texture property, which are the key ingredients to face recognition, are reserved while gradientfaces was used to improve the high frequency component of face images. The effectiveness of illumination process to face image can be appraised through face recognition rate.

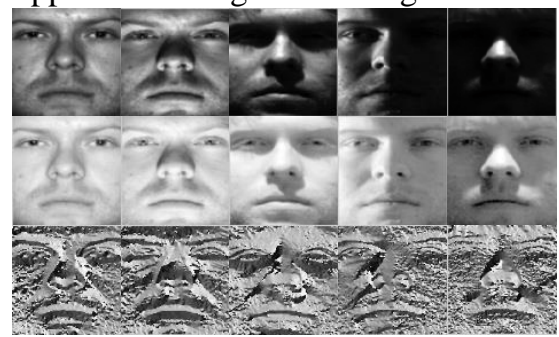

Fig. 3. Face image processed with different method. The first line are original images, the second row are face images processed with nonlinear dynamic range adjustment, the third row are face images processed by our methods.

In order to compare the result with important literature, we show different face recognition rate with different method in Table 2 . The nonlinear dynamic range adjustment (NDRA) and Gradientfaces (GF) have same experiment condition with our experiment. The average face recognition rate is $78.25 \%$, 
99.3\%, 99.45\%, 91.8\%, 98.25\%, 99.1\% and 99.74\% in NDRA, GF, DCT [8], QIR [9], VU [10], OLHE [11] and our method, respectively.

Table. 2. Face recognition rate with different methods on Yale-B database $(\%)$

\begin{tabular}{|c|c|c|c|c|c|c|c|}
\hline Method & NDRA & GF & DCT & QIR & VU & OLHE & $\begin{array}{c}\text { Our } \\
\text { method }\end{array}$ \\
\hline Average & 78.25 & 99.3 & 99.45 & 91.8 & 98.25 & 99.1 & 99.74 \\
\hline
\end{tabular}

\section{Summary}

In this article, we demonstrate a novel method based on the nonlinear dynamic range adjustment and gradientfaces to process face image under varying lighting condition. Firstly, face image was transformed into logarithm domain, in which the grayscale was adjusted by nonlinear dynamic range adjustment using hyperbolic sine function. Secondly, the gradientfaces was adopted to enhance the high frequency detail component of face image and the illumination insensitive was extracted from face image in gradient domain. Experiment results on Yale-B database indicate that the proposed method is robust to varying illumination. We will test the validity of this method in bigger face database in the future.

\section{Acknowledgement}

This research was financially supported by the National Natural Science Foundation of China (NO.61602072) and the Project Foundation of Chongqing Municipal Education Committee (KJ121114).

\section{References}

1. Pai A G, Fernandes S L, Nayak K, et al, Recognizing human faces under varying degree of Illumination: A comprehensive survey, International Conference on Electronics and Communication Systems. Coimbatore, IEEE, 577-582(2015).

2. Shah J H, Sharif M, Raza M, et al. Robust Face Recognition Technique under Varying Illumination[J]. Journal of Applied Research \& Technology, 13 (1):97-105(2015).

3. Nikan S, Ahmadi M, Local gradient-based illumination invariant face recognition using local phase quantisation and multi-resolution local binary pattern fusion. In IET Image Processing, 9(1):12-21(2015).

4. Faraji M R, Qi X. Face recognition under varying illumination based on adaptive homomorphic eight local directional patterns [J]. Iet Computer Vision, 9(3):390-399(2015). 
5. Ming $\mathrm{Y}$, Ruan $\mathrm{Q}, \mathrm{Li} \mathrm{X}$. 3D face reconstruction using a single $2 \mathrm{D}$ face image $[\mathrm{C}] / /$ International Conference on Educational and Information Technology. IEEE, V3-32 - V3-36(2010).

6. Manolovay A, Tonchev K, Boumbarov O, et al. Recognition of facial images with subspace projection and dissimilarity representation[C]// IEEE 6th International Conference on Intelligent Data Acquisition and Advanced Computing Systems: Technology and Applications, (IDAACS),Volume 1, IEEE, 444-449(2011).

7. T Zhang, Y Y Tang, B Fang. et al, Face recognition under varying illumination using gradientfaces, IEEE Transactions on image processing, 18(11): 2599-2606(2009).

8. Chen W, Meng J E, Wu S, Shiqian. W, Illumination compensation and normalization for robust face recognition using discrete cosine transform in logarithm domain. IEEE Transactions on Systems, Man, and Cybernetics, Part B: Cybernetics, 36(2): 458-466(2006).

9. Shan S, Gao W, Cao B, et al. Illumination Normalization for Robust Face Recognition Against Varying Lighting Conditions[C]// IEEE International Workshop on Analysis and Modeling of Faces and Gestures.157-157(2003).

10. $\mathrm{Vu} \mathrm{N} \mathrm{S,} \mathrm{Caplier} \mathrm{A.} \mathrm{Illumination-robust} \mathrm{face} \mathrm{recognition} \mathrm{using} \mathrm{retina}$ modeling[C]// IEEE International Conference on Image Processing. IEEE, 3289-3292(2009).

11. P. Lee, S. Wu and Y Hung, Illumination Compensation Using Oriented Local Histogram Equalization and its Application to Face Recognition, IEEE Transactions on image processing. Volume 21, 4290-4289(2012). 